\title{
Potential Drug Interaction between Warfarin and Linezolid
}

\author{
Yoshiro Sakai ${ }^{1,2}$, Tetsuya Naito ${ }^{1}$, Chiyoko Arima ${ }^{1}$, Miho Miura ${ }^{3}$, Liang Qin ${ }^{2}$, \\ Hidenobu Hidaka $^{2,3}$, Kenji Masunaga ${ }^{2,3}$, Tatsuyuki Kakuma ${ }^{4}$ and Hiroshi Watanabe ${ }^{2,3}$
}

\begin{abstract}
Objective Warfarin is known to interact with many drugs; however, there are currently no descriptions of an interaction with linezolid in the literature. It was recently brought to our attention, however, that several warfarin-medicated patients have experienced an increase in the prothrombin time international normalized ratio (PT-INR) following the administration of linezolid. We therefore performed a retrospective survey in order to investigate the possibility of an interaction between warfarin and linezolid.

Methods The survey items included age, gender, underlying disease, type of surgery, type of infectious disease, duration of linezolid administration, laboratory values and the dose of warfarin. The PT-INR was observed over time before treatment and at days 4 or 5 and 10, completion and one week after the end of concomitant therapy.

Patients The subjects included six patients who were recovering from recent heart-related surgery.

Results The PT-INR increased from $1.62 \pm 0.32$ before concomitant linezolid administration to $3.00 \pm 0.83$ at day 4 or 5 after concomitant administration $(\mathrm{p}<0.01)$ and significantly decreased from $1.65 \pm 0.45$ at the completion of the regimen to $1.26 \pm 0.1$ one week later $(\mathrm{p}<0.05)$. With respect to the relationship between the dose of warfarin and the PT-INR in five cases, the PT-INR increased following concomitant linezolid treatment in all cases.

Conclusion Although it has been reported that linezolid does not influence the metabolism or protein binding of warfarin, our data showed potential drug interactions between warfarin and linezolid. Our data suggest that PT-INR monitoring after the completion of concomitant warfarin and linezolid therapy is important.
\end{abstract}

Key words: warfarin, linezolid, drug interaction, PT-INR

(Intern Med 54: 459-464, 2015)

(DOI: 10.2169/internalmedicine.54.3146)

\section{Introduction}

Warfarin does not directly act on coagulation factors present in the circulation, but rather exerts anticoagulant and thrombus formation-preventing effects by inhibiting the protein synthesis of vitamin K-dependent coagulation factor in the liver, and is used to treat and prevent both myocardial infarction and pulmonary embolism (1). However, warfarin is known to interact with various drugs, although the mechanism of interaction remains unclear in many cases, and not all drug combinations have been investigated. Therefore, it is necessary to pay close attention to changes in the pa- tient's coagulative ability when concomitantly adding or withdrawing other drugs during anticoagulant therapy (2).

Linezolid is an oxazolidinone antibiotic that displays an antimicrobial activity against Gram-positive cocci, including resistant bacteria, such as methicillin-resistant Staphylococcus aureus (MRSA) (3). Linezolid exhibits a superior capacity for tissue transfer (4) and is expected to be efficacious in cases of infections that were previously difficult to treat due to problems in drug transfer. Linezolid is afforded an important status in the treatment guidelines for MRSA infection established by the Infectious Diseases Society of America (IDSA) in February 2011 (5).

Warfarin reportedly interacts with many antibiotics, as de-

\footnotetext{
${ }^{1}$ Department of Pharmacy, Kurume University Hospital, Japan, ${ }^{2}$ Department of Infection Control and Prevention, Kurume University School of Medicine, Japan, ${ }^{3}$ Division of Infection Control and Prevention, Kurume University Hospital, Japan and ${ }^{4}$ Biostatistics Center, Kurume University School of Medicine, Japan

Received for publication April 22, 2014; Accepted for publication July 13, 2014

Correspondence to Dr. Yoshiro Sakai, sakai_yoshirou@kurume-u.ac.jp
} 
Table 1. Patient Background

\begin{tabular}{|c|c|c|c|c|c|c|c|c|c|}
\hline $\begin{array}{l}\text { Case } \\
\text { number }\end{array}$ & Age & Gender & $\begin{array}{l}\text { Underlying } \\
\text { disease }\end{array}$ & $\begin{array}{l}\text { Body weight } \\
(\mathrm{kg})\end{array}$ & Surgery & $\begin{array}{l}\text { Infectious } \\
\text { disease }\end{array}$ & $\begin{array}{l}\text { Causative } \\
\text { organism }\end{array}$ & $\begin{array}{l}\text { Linezolid starting } \\
\text { day }\end{array}$ & $\begin{array}{l}\text { Duration of linezolid } \\
\text { administration (day) }\end{array}$ \\
\hline Case 1 & 63 & Male & $\begin{array}{l}\text { Mitral } \\
\text { insufficiency }\end{array}$ & 64.2 & Valvoplasty & $\begin{array}{l}\text { Infectious } \\
\text { endocarditis }\end{array}$ & MRSA & $\begin{array}{l}13 \text { days } \\
\text { postoperation }\end{array}$ & 50 \\
\hline Case 2 & 40 & Male & $\begin{array}{l}\text { Mitral } \\
\text { insufficiency }\end{array}$ & 67.7 & $\begin{array}{l}\text { Valve replacement } \\
\text { (artificial valve) }\end{array}$ & Graft infection & Unclear & $\begin{array}{l}\text { about } 10 \text { years } \\
\text { after the surgery }\end{array}$ & 19 \\
\hline Case 3 & 78 & Male & $\begin{array}{l}\text { Mitral } \\
\text { insufficiency }\end{array}$ & 52.7 & $\begin{array}{l}\text { Valve replacement } \\
\text { (biological valve) }\end{array}$ & $\begin{array}{l}\text { Infectious } \\
\text { endocarditis }\end{array}$ & $\begin{array}{l}\text { Streptococcus } \\
\text { constellatus }\end{array}$ & $\begin{array}{l}8 \text { days } \\
\text { postoperation }\end{array}$ & 9 \\
\hline Case 4 & 62 & Female & $\begin{array}{l}\text { Mitral } \\
\text { insufficiency }\end{array}$ & 46.1 & Valvoplasty & $\begin{array}{l}\text { Infectious } \\
\text { endocarditis }\end{array}$ & MRSE & $\begin{array}{l}21 \text { days } \\
\text { postoperation }\end{array}$ & 33 \\
\hline Case 5 & 69 & Male & $\begin{array}{l}\text { Acute aortic } \\
\text { dissection }\end{array}$ & 77.6 & Root replacement & Mediastinitis & MRSE & $\begin{array}{l}14 \text { days } \\
\text { postoperation }\end{array}$ & 25 \\
\hline Case 6 & 59 & Female & $\begin{array}{l}\text { Mitral } \\
\text { insufficiency }\end{array}$ & 59.3 & $\begin{array}{l}\text { Valve replacement } \\
\text { (artificial valve) }\end{array}$ & $\begin{array}{l}\text { Infectious } \\
\text { endocarditis }\end{array}$ & $\alpha$-Streptococcus & $\begin{array}{l}3 \text { days } \\
\text { preoperation }\end{array}$ & 28 \\
\hline
\end{tabular}

scribed above, and attention must be paid to the potential for hemorrhagic complications (6). However, there are currently no descriptions of an interaction between warfarin and linezolid on the interview forms for either drug $(2,3)$. We encountered several patients who experienced an increase in the prothrombin time international normalized ratio (PT-INR) while receiving simultaneous treatment with warfarin and linezolid after cardiovascular surgery. We thus retrospectively surveyed patients who had simultaneously been treated with warfarin and linezolid and investigated the possibility of drug interactions based on changes in the PT-INR values, concomitantly administered drugs and/or the dose of warfarin in order to clarify possible interactions between these two drugs.

\section{Materials and Methods}

\section{Ethical approval}

All studies described herein were approved by the Human Ethics Review Board of Kurume University (13094).

\section{Survey period and subjects}

The subjects included patients who has simultaneously been treated with warfarin and linezolid between January and December, 2011 at Kurume University Hospital. Patients already being treated with drugs known to influence the PT-INR when concomitantly administered with warfarin were excluded from the study.

\section{Survey items and methods}

In order to investigate the occurrence of drug interactions in patients simultaneously treated with warfarin and linezolid, the subjects' electronic medical records were retrospectively surveyed. The survey items included age, gender, body weight, underlying disease, type of surgery, type of infectious disease, duration of linezolid administration, laboratory values [aspartate aminotransferase (AST), alanine aminotransferase (ALT), blood urea nitrogen (BUN), serum creatinine (Scr), serum albumin (Alb) and PT-INR], the dose of warfarin and concomitantly administered drugs. The labo- ratory values, excluding the PT-INR, were compared with respect to the following four time points of concomitant administration: before treatment, during treatment (at the time of the highest PT-INR), at the completion of treatment and one week after the completion of treatment. The PT-INR was observed over time before therapy and at days 4 or 5 and 10 , completion and one week after the completion of concomitant therapy. There was a change in the measurement protocol for determining the PT-INR after a few years. Therefore, a post-surgery group that did not receive concomitant treatment with linezolid for mitral insufficiency was investigated for comparison purposes.

We defined the start date of linezolid therapy as day 0 and investigated changes in the dose of warfarin and the PTINR from before treatment to five days after treatment.

\section{Statistical analysis}

The laboratory data are presented as the mean \pm standard deviation, and were compared using a paired t-test, with the significance level set at less than 5\%.

The JMP10 software program (SAS Institute, Inc., Cary, USA) was used for the statistical analysis.

\section{Results}

Six of the seven patients who had been simultaneously treated with warfarin and linezolid during the survey period were investigated in detail. One patient was excluded due to being previously treated with rifampicin, which reduces the effect of warfarin. That patient's background characteristics are shown in Table 1. The mean age of the six patients was $61.8 \pm 12.6$ years. There were four men and two women, with a body weight of $61.3 \pm 11.2 \mathrm{~kg}$, all of whom had undergone heart-related surgery. Linezolid was indicated for heartrelated infection, and resistant bacteria were detected as the etiologic microorganism in half of these cases, with the most infectious form, endocarditis, being detected in four cases. The administration of anticoagulant therapy for at least three months is necessary after surgery for valve replacement and valvuloplasty; therefore, treating the infectious disease was also important, although we were careful 
Table 2. Concomitant Warfarin and Linezolid Administration-induced Changes in Laboratory Test Values

\begin{tabular}{|c|c|c|c|c|}
\hline & Before concomitant administration & During concomitant administration & At completion of concomitant administration & 1 week after completion \\
\hline AST & $25.2 \pm 7.36$ & $26.2 \pm 4.88$ & $18.8 \pm 9.09$ & $18.8 \pm 6.43$ \\
\hline ALT & $27.8 \pm 7.6$ & $31.8 \pm 10.6$ & $26.3 \pm 18.1$ & $22.2 \pm 21.8$ \\
\hline BUN & $17.1 \pm 5.01$ & $13.2 \pm 5.27$ & $15.2 \pm 4.59$ & $17.8 \pm 5.75$ \\
\hline Scr & $0.87 \pm 0.31$ & $0.83 \pm 0.26$ & $0.93 \pm 0.21$ & $0.85 \pm 0.27$ \\
\hline Alb & $2.75 \pm 0.39$ & $2.87 \pm 0.20$ & $3.39 \pm 0.47$ & $3.68 \pm 0.59$ \\
\hline
\end{tabular}
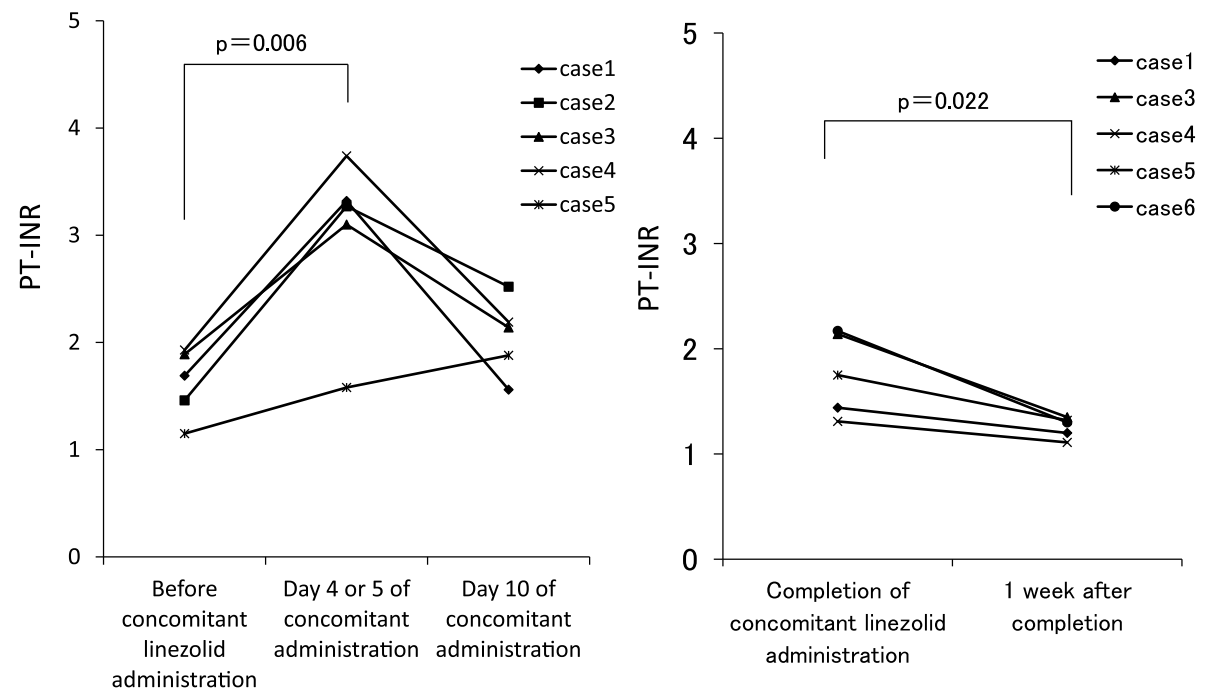

Figure 1. Changes in the PT-INR values during and at the completion of concomitant warfarin and linezolid administration. The PT-INR values significantly increased from $1.62 \pm 0.32$ before concomitant linezolid administration to $3.00 \pm 0.83$ on day 4 or 5 of concomitant treatment $(p<0.01)$ and decreased on day 10 due to dose reduction or the withdrawal of warfarin and the concomitant administration of rifampicin. The administration of warfarin was discontinued for surgery after the completion of the concomitant therapy and switched to vitamin $K$ in one patient. Excluding this patient, the PT-INR values significantly decreased from $1.65 \pm 0.45$ at the completion of treatment to $1.26 \pm 0.1$ at one week after the completion of treatment $(p<0.05)$.

to monitor for a bleeding tendency in the four patients who required warfarin despite it being contraindicated in cases involving treatment for infectious endocarditis. Linezolid was started postoperatively in five cases. The mean duration of linezolid administration was $27.3 \pm 13.8$ days, and the dose of linezolid was $600 \mathrm{mg}$ IV every 12 hours in all patients. The infectious diseases improved following the administration of linezolid.

The liver and renal functions were in the normal range at the time of initiation of concomitant warfarin and linezolid therapy in all cases. The changes in the laboratory values, excluding PT-INR, are shown in Table 2. Consequently, concomitant warfarin and linezolid treatment did not induce significant changes in any of the following indicators: AST, ALT, BUN, Scr, BUN or Alb. In addition, no other concomitant drugs were administered before the maximum PTINR was reached after the initiation of concomitant linezolid and warfarin in any of the patients, and no other concomitant drugs were administered one week after the completion of the concomitant therapy.

The changes in the PT-INR values are shown in Figs. 1 and 2. There were no significant changes in the PT-
INR values three days before or on the day of initiation of concomitant treatment, and the dose of warfarin was not changed in any of the patients, with the exception of one case in which the warfarin dose was increased from 4 to 4.5 mg. In Case 6, treatment with linezolid was started with the administration of warfarin. Excluding this patient, the PTINR values significantly increased from $1.62 \pm 0.32$ before concomitant linezolid administration to $3.00 \pm 0.83$ on day 4 or 5 of concomitant therapy $(\mathrm{p}<0.01)$ and then decreased by day 10 due to dose reduction or the withdrawal of warfarin and the concomitant administration of rifampicin. No bleeding tendency, as assessed according to the results of PT-INR monitoring, was observed in any of the patients, either after dose reduction or the withdrawal of warfarin following the concomitant administration of warfarin and linezolid. In Case 2, the administration of warfarin was discontinued for surgery after the completion of the concomitant therapy and the drug regimen was switched to vitamin K. Excluding this patient, the PT-INR values significantly decreased from $1.65 \pm 0.45$ at completion to $1.26 \pm 0.1$ at one week after completion $(\mathrm{p}<0.05)$.

Fig. 2 shows the relationship between the dose of war- 

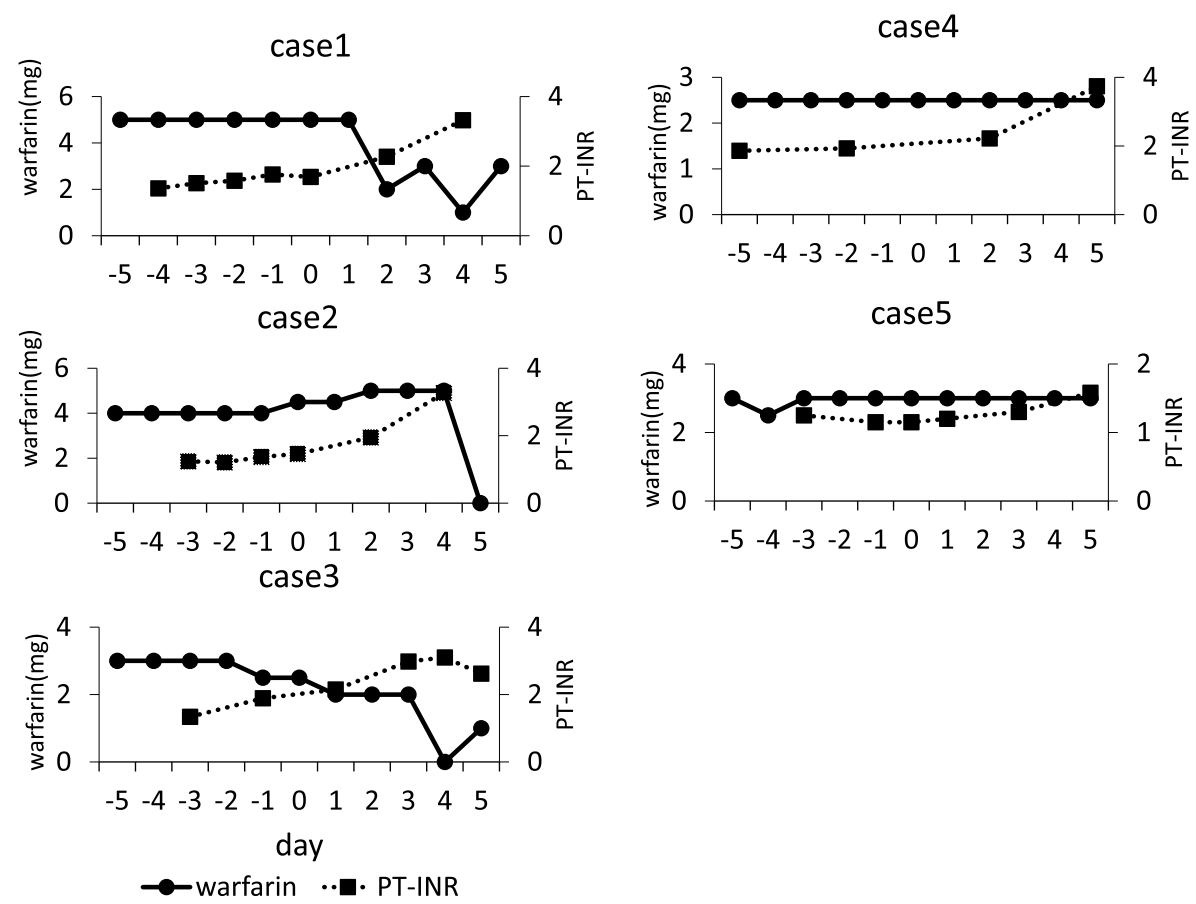

Figure 2. Relationship between the dose of warfarin and the PT-INR in the five patients. We defined the start date of linezolid treatment as day 0 and investigated changes in the dose of warfarin and the PT-INR before and after the administration of concomitant therapy for five days. Consequently, the PT-INR values increased during concomitant linezolid administration in all cases. In Cases 1, 2 and 3, the dose of warfarin was reduced or withdrawn after the concomitant administration of warfarin and linezolid. In Case 4, the dose of warfarin was not changed. In Case 5, the dose of warfarin was not changed, except for day $\mathbf{- 4}$.

farin and the PT-INR in the five cases. The PT-INR values increased during the concomitant administration of linezolid in all cases. In Cases 1, 2 and 3, the dose of warfarin was reduced or withdrawn following the concomitant administration of warfarin and linezolid. In Case 4, the dose of warfarin was not changed. In Case 5, the dose of warfarin was only changed on day -4 .

The administration of concomitant warfarin and linezolid was started on days $8,13,14$ and 21 in the postoperative cases. In order to determine the changes in the PT-INR values in the cases treated without linezolid, we investigated the PT-INR and dose of warfarin from days 8 through 28 after surgery for mitral insufficiency. The control group included four patients, with a mean age of 74.3 \pm 5.7 years. Fig. 3 shows the relationship between the dose of warfarin and the PT-INR in this group. The PT-INR increased slowly in control Case 1, although this increase was not as high as that observed among the patients treated with concomitant linezolid. Meanwhile, the PT-INR values changed when the dose of warfarin was changed in control Cases 2 and 3. In control Case 4, a decrease in the dose of warfarin resulted in a simultaneous decrease in the PT-INR.

\section{Discussion}

There are some caveats to interpreting the results of the statistical analyses. Because the available sample size was small, our data analysis should be used only to generate hypotheses, not as a confirmatory analysis.

In previous studies, the PT-INR values decreased when warfarin and linezolid were simultaneously administered in healthy adults, although the decrease was not clinically problematic (7). In contrast, our data analysis showed a tendency toward an increase in the PT-INR when the dose of warfarin was decreased. We speculated that the changes in the postoperative PT-INR values in the control group were influenced by the dose of warfarin. However, the dose of warfarin was increased prior to the administration of concomitant warfarin and linezolid in only one case. Therefore, we do not believe that the increase in the PT-INR values was influenced by surgery. In addition, we speculated that the increase in the PT-INR values was perhaps due to the effects of infectious disease. However, although the degree of inflammatory reactions observed before the concomitant administration of linezolid was high, the PT-INR values only increased when the inflammatory reactions decreased. Therefore, we do not consider the rise in the PT-INR values to be the result of infection. These results suggest the possible presence of a drug interaction between warfarin and linezolid.

Patients with liver and kidney disease and hypoalbuminemia are reportedly at risk of hemorrhage when being treated with warfarin $(8,9)$. However, no significant signs of hemorrhage were reported after the concomitant administration 

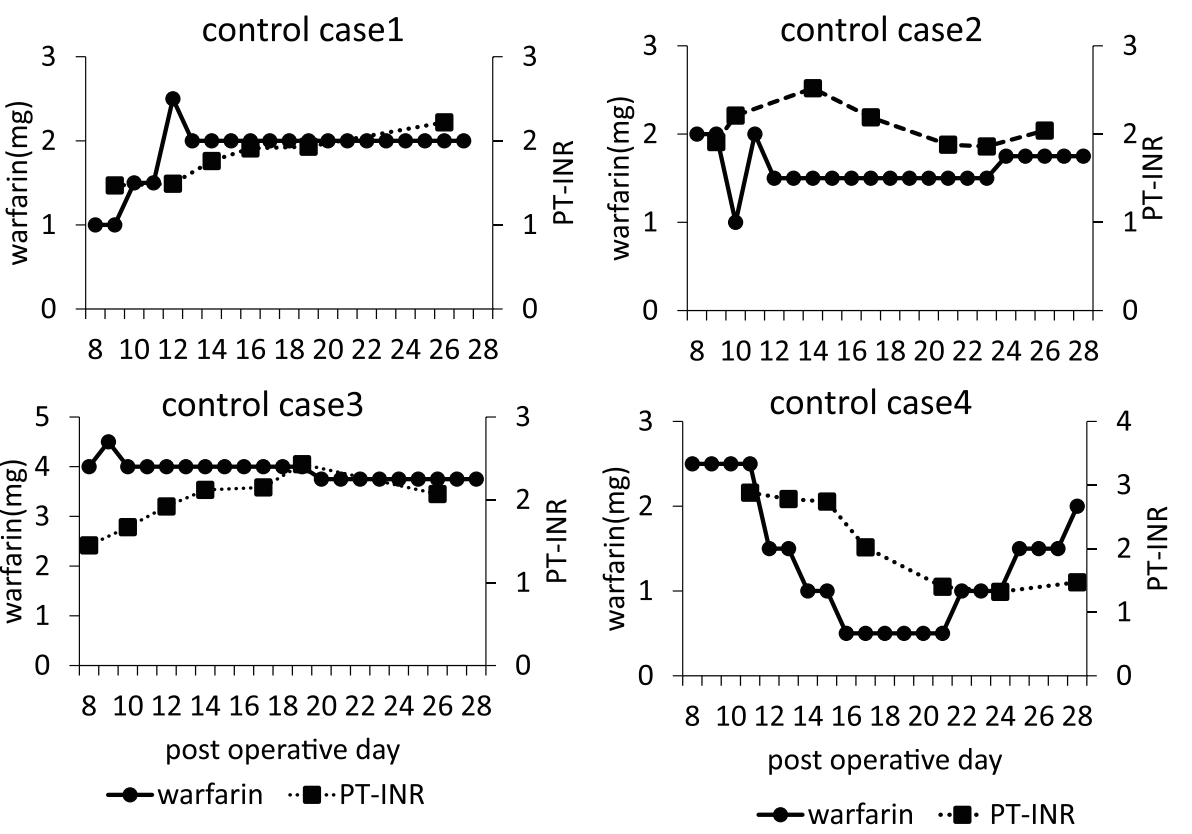

Figure 3. Relationship between the dose of warfarin and the PT-INR among the four subjects in the control group. We defined the date of surgery as day 0 and investigated changes in the dose of warfarin and the PT-INR from days 8 through 28. Consequently, the PT-INR values increased slowly in control Case 1, although the rise in the PT-INR was not as high as that observed among the patients who received concomitant linezolid administration. Meanwhile, the PT-INR values changed when the dose of warfarin was changed in control Cases 2 and 3. In control Case 4, a decrease in the dose of warfarin resulted in a simultaneous decrease in the PT-INR.

of linezolid in this study, suggesting that these diseases did not affect our subjects' laboratory values. Linezolid is reportedly mostly metabolized in the tissues of healthy adults, with the main site of metabolism being the liver (3). The metabolism of linezolid has been shown to not be affected by cytochrome $\mathrm{P} 450$, and this drug is believed to be primarily oxidized non-enzymatically by reactive oxygen species distributed throughout the body (10). Therefore, the interaction observed between warfarin and linezolid in this study may not have been due to metabolism by the cytochrome system.

In addition to the actions of metabolic enzymes, drug interactions with warfarin are believed to occur via substitutions for protein binding and involvement with the effects of vitamin $\mathrm{K}(2)$. The rate of protein binding of linezolid is low (31\%) (3), which reflects its superior capacity for tissue transfer. Since linezolid is rapidly transferred to the tissue after administration, an interaction between warfarin and linezolid via protein binding in the circulation is unlikely. In the present survey, the changes in the albumin levels from before the initiation of concomitant treatment with linezolid and warfarin to the time of the highest PT-INR measurement were not significant, and the PT-INR values increased after the concomitant administration of the two drugs; therefore, the effects of protein binding were minimal. Some antibiotics reportedly strengthen the effects of warfarin by decreasing the levels of intestinal vitamin K-producing bacteria. Similarly, it has been reported that linezolid possesses an antibacterial activity for Bifidobacterium producing vitamin
$\mathrm{K}$ (11). Hence, we considered that linezolid may potentiate the effects of warfarin by lowering the level of vitamin $\mathrm{K}$, thus increasing the PT-INR.

The difficulty in managing the coagulation system under concomitant therapy with rifampicin and warfarin is well documented (12). Our survey found that the PT-INR reaches a maximum on day 4 of concomitant warfarin and linezolid administration in early cases and day 20 in late cases, suggesting that it is also difficult to manage the PT-INR during the concomitant administration of these drugs and that periodic monitoring of the coagulation system is required.

In the present study, we demonstrated the possibility for an interaction between warfarin and linezolid in patients undergoing cardiovascular surgery. In this study, warfarin was administered to prevent postoperative thromboembolism in all patients. Warfarin is also used in non-postoperative patients and is indicated for the prevention of atrial fibrillation-associated thromboembolism. In future studies, the survey period should be extended and the number of cases and targets should be increased. In addition, differences in the indications for warfarin therapy should be investigated. It is also necessary to clarify the mechanism of interaction between these two drugs. Based on a review of the literature, we speculate that linezolid increases the actions of warfarin by lowering the vitamin $\mathrm{K}$ level.

The coagulation system must be monitored by measuring the PT-INR in order to determine the need to change the dose of warfarin when concomitantly administering linezolid. Sufficient monitoring is also necessary after the com- 
pletion of concomitant treatment.

This article concerns the content of a study that attracted the attention of the manager of the fourth Japan Society of Chemotherapy, West Japan Branch Activation Committee Special Prize.

The authors state that they have no Conflict of Interest (COI).

\section{References}

1. Davie EW, Fujikawa K, Kisiel W. The coagulation cascade: initiation, maintenance, and regulation. Biochemistry 30: 10363-10370, 1991.

2. Eisai Co. Interview form. Warfarin Tablets. [Internet]. [cited 2014 Mar 25]. Available from: http://www.info.pmda.go.jp/

3. Pfizer Co. Interview form. Zyvox Injection. [Internet]. [cited 2014 Mar 25]. Available from: http://www.info.pmda.go.jp/

4. Wiskirchen DE, Shepard A, Kuti JL, et al. Determination of tissue penetration and pharmacokinetics of linezolid in patients with diabetic foot infections using in vivo microdialysis. Antimicrob Agents Chemother 55: 4170-4175, 2011.

5. Liu C, Bayer A, Cosgrove SE, et al. Infectious Diseases Society of America.Clinical practice guidelines by the infectious diseases society of america for the treatment of methicillin-resistant Staphylococcus aureus infections in adults and children. Clin In- fect Dis 52: e18-e55, 2011.

6. Baillargeon J, Holmes HM, Lin YL, et al. Concurrent use of warfarin and antibiotics and the risk of bleeding in older adults. Am J Med 125: 183-189, 2012.

7. Stalker DJ, Jungbluth GL. Clinical pharmacokinetics of linezolid, a novel oxazolidinone antibacterial. Clin Pharmacokinet 42: 11291140, 2003.

8. Abdelhafiz AH, Myint MP, Tayek JA, et al. Anemia, hypoalbuminemia, and renal impairment as predictors of bleeding complications in patients receiving anticoagulation therapy for nonvalvular atrial fibrillation: a secondary analysis. Clin Ther 31: 1534-1539, 2009.

9. DiMarco JP, Flaker G, Waldo AL, et al; AFFIRM Investigators. Factors affecting bleeding risk during anticoagulant therapy in patients with atrial fibrillation: observations from the Atrial Fibrillation Follow-up Investigation of Rhythm Management (AFFIRM) study. Am Heart J 149: 650-656, 2005.

10. Slatter JG, Stalker DJ, Feenstra KL, et al. Pharmacokinetics, metabolism, and excretion of linezolid following an oral dose of [(14)C]linezolid to healthy human subjects. Drug Metab Dispos 29: 1136-1145, 2001.

11. Moubareck C, Gavini F, Vaugien L, et al. Antimicrobial susceptibility of bifidobacteria. J Antimicrob Chemother 55: 38-44, 2005.

12. Lee CR, Thrasher KA. Difficulties in anticoagulation management during coadministration of warfarin and rifampin. Pharmacotherapy 21: 1240-1246, 2001.

(C) 2015 The Japanese Society of Internal Medicine http://www.naika.or.jp/imonline/index.html 